\title{
Mobile Crowdsensing Services for Tinnitus Assessment and Patient Feedback
}

\author{
Rüdiger Pryss ${ }^{1}$, Winfried Schlee ${ }^{2}$, Berthold Langguth ${ }^{2}$, Manfred Reichert ${ }^{1}$ \\ ${ }^{1}$ Institute of Databases and Information Systems, Ulm University, Germany \\ ${ }^{2}$ Clinic and Policlinic for Psychiatry and Psychotherapy, University of Regensburg, Germany \\ 1 \{ruediger.pryss, manfred.reichert $\} @$ uni-ulm.de \\ winfried.schlee@gmail.com, berthold.langguth@medbo.de
}

\begin{abstract}
Assessment of chronic disorders requires new ways of data collection compared to the traditional pen \& paper based approaches. For example, tinnitus, the phantom sensation of sound, is a highly prevalent disorder that is difficult to treat; i.e., available treatments are only effective for patient subgroups. In most individuals with tinnitus, loudness and annoyance of tinnitus varies over time. Currently, established assessment methods of tinnitus neither systematically assess this moment-to-moment variability nor environmental factors having an effect on tinnitus loudness and distress. However, information of individual fluctuations and the effect of environmental factors on the tinnitus might represent important information for tinnitus subtyping and for individualized treatment. In this context, a promising approach for collecting ecological valid longitudinal datasets at rather low costs is mobile crowdsensing. In the TrackYourTinnitus project, we developed an advanced mobile crowdsensing platform to reveal more detailed information about the course of tinnitus over time. In this paper, the patient mobile feedback service as a particular component of the platform is presented. It was developed to provide patients with aggregated information about the variation of their tinnitus over time. This mobile feedback service shall help a patient to demystify the tinnitus and to get better control of it, which should facilitate coping with this chronic health condition. As the basic principles and design of this mobile services are also applicable to other chronic disorders, promising perspectives for disorder management and clinical research arise.
\end{abstract}

Keywords-Mobile Crowdsensing, Mobile Healthcare Application, Patient Feedback, Mobile Healthcare Service, Mobile Service.

\section{INTRODUCTION}

Healthcare craves for new ways of collecting large and ecological valid longitudinal data. This applies to the assessment of tinnitus as well. Tinnitus is a highly prevalent disorder, for which currently no sufficient therapy exist [1]. Furthermore, Tinnitus is a purely subjective sensation that can only be assessed by the report of the individual patient. The pathophysiology of tinnitus is incompletely understood and clinical trials frequently reveal contradictory results. Presumably, these non-conclusive results can be explained by the fact that tinnitus is not a homogeneous clinical entity. Instead, there exist many forms of tinnitus, varying in their clinical characteristics as well as in the response to specific therapeutic interventions [2], [3]. Additional complexity is introduced by the fact that the perception of tinnitus loudness and distress is not constant in most cases, but varies over time depending on the context (e.g., environmental sound level or stress) [4].

Currently, tinnitus is assessed based on questionnaires, visual analogue scales or psychoacoustic measurements. However, these assessment methods, which are used both in clinical practice and research, do not capture the withinday and between-day variability of tinnitus loudness and distress over time. Moreover, contextual and environmental influence on Tinnitus loudness questions the current routine, where assessments are performed in most cases in clinics or at home, but practically never during work or any other activity of daily life. In order to mitigate these shortcomings, new ways of collecting ecological valid longitudinal datasets at rather low costs from patients during their daily life are required. For this purpose, we developed the mobile crowdsensing platform TrackYourTinnitus (TYT). The latter tracks individual tinnitus perception using smart mobile devices of users. The tracking procedure comprises a specific questionnaire we developed to assess tinnitus perception and tinnitus-related parameters during the daily routine of a user. Additionally, the smart mobile device of a user records the environmental sound level, while the user fills in the assessment questionnaire. Results are transferred to the TYT backend that, in turn, offers features enabling researchers to evaluate gathered patient data. Note that in the context of personalized healthcare, mobile crowdsensing offers completely new perspectives [4]-[7] on the daily routine of patients.

The analysis of the first data assessed with TYT [4], [8], [9] has confirmed the hypotheses of (1) a relevant variability of tinnitus loudness and annoyance for the majority of patients and (2) an interaction with exogenous and endogenous factors. These findings have high relevance for individual patients: The TYT may detect specific relationships between influencing factors and tinnitus annoyance, which have not been identified by patients in conventional studies before. For example, tinnitus annoyance may depend on the stress level the patient had the day before. Information of the patients about such relationships may (1) help gaining more control about a symptom that seemed to be completely uncontrollable, (2) provide guidance for behavior and thus help to better cope with tinnitus and perceive the tinnitus as less 
stressful. Moreover, smart feedback on tinnitus variability and influencing factors is expected to motivate users to use the mobile TYT services.

Such results highlight the potential of Ecological Momentary Assessment (EMA; also known as: ambulatory assessment \& experience sampling), which is provided by TYT, to support clinicians in assessing neuropsychiatric symptoms accurately and in making valid diagnoses. In EMA, the variable in question (e.g., symptoms) is assessed repeatedly in daily life [10]. Instead of retrospectively asking the individuals (in an interview or questionnaire) how strong they experienced a symptom in a given past time interval, the individuals are asked how they currently experience the symptom; this is done at several time points within the given time interval.

This paper presents the mobile feedback service of the TYT platform. We provide detailed backgrounds, present technical issues, and discuss the perspective of patients on the feedback service. In this context, the developed feedback service is expected to increase general user motivation. The remainder of this paper is organized as follows: Section II introduces the TYT platform and its main features. In Section III, the mobile service for patient feedback is presented. Finally, Section IV discusses related work and Section V concludes the paper with a summary and outlook.

\section{The Track YourTinnitus PlatForm}

The TYT mobile crowdsensing platform aims at measuring fluctuations of tinnitus perception and tinnitus distress under real life conditions during the patient's day. In particular, mobile crowdsensing services shall enable researchers to gather data from huge numbers of users. Note that this allows tracking the moment-to-moment fluctuation of the tinnitus. Furthermore, tracked data may be related to everyday behavior as well as the daily routine of patients to systematically identify relationships between individual routines and tinnitus fluctuations. Moreover, the TYT mobile crowdsensing platform can be further developed to assess the effects of specific standardized therapeutic interventions.

We developed the TYT mobile crowdsensing platform as a multidisciplinary research team consisting of psychologists, physicians, and computer scientists. The platform comprises a website for user registration, two mobile applications (for iOS and Android), and a MySQL database as a central repository for the data collected [6], [11], which can be made available to the clinicians and researchers. The website also provides two important other features: (1) users can visualize their recorded tinnitus data and (2) users can provide information about their current tinnitus treatment. In order to be able to track the daily tinnitus perception, the following procedure must be accomplished by a user.

First, users have to create an TYT account using our website.
Second, after registering, users have to fill in three registration questionnaires. First, users have to fill in the "MiniTQ-12" questionnaire, which measures tinnitus-related psychological problems. Second, users have to fill in the "Tinnitus Sample Case History Questionnaire (TSCHQ)". The TSCHQ questionnaire determines the current tinnitus status of the user as well as his tinnitus history. Finally, users have to fill in the "Worst Symptom" questionnaire. This questionnaire asks the user about his current worst symptom caused by tinnitus. While the first two questionnaires constitute already used instruments, the third one have been newly developed by the authors. Altogether, users have to complete 58 questions with respect to the three questionnaires. The completion of these three questionnaires is a prerequisite to be able to use the TYT website features as well as the mobile applications.

Third, after the registration questionnaires have been completed, a user can use the mobile applications to track the daily tinnitus perception. Therefore, the user has to log in to the Android or iOS mobile application. Then, he is asked to fill in the assessment questionnaire developed by us. The questionnaire comprises 8 questions (cf. Table I) and rates the tinnitus perception of the user when being asked (e.g., current tinnitus loudness).

Fourth, the assessment questionnaire, in turn, is provided in two ways: (1) the mobile application automatically applies the questionnaire to the user or (2) the user makes the conscious decision to fill in the questionnaire. The first way is our desired procedure and realized as follows: The assessment questionnaire is randomly presented to the user up to 12 times per day. Therefore, we realized a notification feature for Android and iOS as well as a notification algorithm [6]. This procedure of applying the assessment questionnaire ensures that (1) users cannot foresee the time of being asked and that (2) users are asked in various daily situations. Such a randomized approach was realized in order to improve the ecological validity of the method applied.

Fifth, while filling in the assessment questionnaire, the smart mobile device of a user records the environmental sound level. Currently, the sound level measurements are evaluated in more detail. One question, among others, that arises is based on the fact whether measurements of the iOS platform and the Android platform are comparable.

Sixth, finally, results gathered with the assessment questionnaire and sound recording are transferred to the TYT database. The latter, in turn, offers features enabling researchers to evaluate gathered patient data. This feature has been used for the results presented in this paper.

\section{PAtient FEEDBACK}

Experiments we had conducted with the TYT platform and its mobile services revealed that proper feedback on the collected data is essential for users in order to increase their motivation for regularly using the mobile app. Note that 


\begin{tabular}{|c|c|c|c|}
\hline & Question & Scale & $\begin{array}{l}\text { Measurement } \\
\text { of }\end{array}$ \\
\hline (1) & Did you perceive the tinnitus right now? & BS & Perception \\
\hline (2) & How loud is the tinnitus right now? & VAS & Loudness \\
\hline (3) & How stressful is the tinnitus right now? & VAS & Strain \\
\hline (4) & How is your mood right now? & VAS & Mood \\
\hline (5) & How is your arousal right now? & VAS & Arousal \\
\hline (6) & Do you feel stressed right now? & VAS & Stress \\
\hline (1) & $\begin{array}{l}\text { How much did you concentrate on the things } \\
\text { you are doing right now? }\end{array}$ & VAS & Concentration \\
\hline (8) & Do you feel irritable right now? & BS & Irritability \\
\hline
\end{tabular}

Table I: TrackYourTinnitus Assessment Questions

proper feedback constitutes a salient incentive for patient engagement in the context of mobile healthcare services in general [12]. Regarding tinnitus, for example, a welldesigned feedback function should provide the patients with information that allows them to better understand the dependencies between tinnitus loudness and annoyance on environmental factors. This information shall help them to demystify tinnitus, to obtain an improved control, and to better cope with their tinnitus. Our results confirm that about 40 percent of the tinnitus variance can be explained with the variance of exogenous and endogenous factors. If individual users have understood this relationship, they can get better control over their tinnitus. Motivated by this data gathered with TYT, we developed a sophisticated mobile feedback service. The latter was integrated with both the TYT backend and the Android mobile application.

In general, different approaches for providing mobile feedback can be distinguished. First of all, feedback could be provided by medical experts based on the information gathered with the smart mobile device. Alternatively, feedback can be automatically generated by smart services and information systems respectively. Furthermore, the way how feedback is provided to users is essential. TYT comprises a mobile feedback service that automatically generates user feedback and additionally provides the option for transferring selected information to the treating physician, who can then give feedback. Whether feedback is based on real time data only or also considers historical data constitutes another differentiation. The TYT service considers historical data gathered with the assessment questionnaire. Based on this data, individual feedback is calculated automatically.

Finally, we learned that the ability to configure parameters relevant for feedback calculation is highly welcome by users. TYT allows them to specify a time window that shall be applied to the personal data gathered with the assessment questionnaire. If a user specifies the respective parameter, feedback calculation will be limited to the specified time window. Therefore, the parameter allows patients to check whether the received feedback has evolved over time.

This section presents the TYT mobile feedback service along three perspectives. First, we sketch the overall feed- back procedure and present factors relevant in this context. Second, we discuss the user perspective on the feedback service. Finally, we present technical issues related to the developed feedback algorithms.

\section{A. Overall Feedback Procedure}

Fig. 1 gives an overview on the mobile feedback service. Its general idea is to categorize patients based on the collected data and to provide specific feedback depending on the category the patient is assigned to. Accordingly, patients are categorized based on their questionnaire data. The categorization of individuals will be calculated automatically. For example, if in a given individual the tinnitus loudness correlates with stress levels, the person will be automatically assigned to category stress.

Altogether, we identified four categories (cf. Fig. 1(5)). The four patient categories are derived based on analyses of the data collected by all TYT users (cf. Fig. 1(2)). Thereby, we focused on the correlation of subjective loudness (cf. Table I, Question 2) with other measurements (cf. Table I, Questions 3-8). If we had observed a particular correlation for a considerable subset of the patients, we derived a corresponding feedback category. This analysis revealed that a correlation with Question 1 is not relevant. Note that Question 1 solely considers the current tinnitus situation. That means, patients may not perceive the tinnitus right now, but perceive it in general. The remaining correlations for strain (cf. Table I, Question 3) and irritability (cf. Table I, Question 8) require further considerations before taking them into account.

Assigning patients to one of the four categories constitutes the first part of the feedback procedure (cf. Fig. 1(5)). Furthermore, each category is coupled with specific interpretations. These interpretations, in turn, are created by medical experts using the TYT backend and include, for example, general recommendations (cf. Fig. 18). If a relationship between perceived stress and perceived tinnitus is detected, for example, the feedback about it further includes the information that there exist specific approaches for stress reduction (cf. Fig. 1(6). These interpretations, in turn, are assigned to one or more of the categories, again with the help of the TYT backend (cf. Fig. 1(6)). Furthermore, interpretations are associated with detailed explanations that will be created by the medical experts as well. Patients may rate these explanations (cf. Fig. 1()) to inform the medical experts whether they have benefited from it. The interpretations together with the explanations constitute the second part of the feedback. Moreover, the two discussed parts form the entire feedback for an individual patient (cf. Fig. 1(4)). Technically, the feedback will be provided by the TYT mobile feedback service.

There are two additional aspects of the TYT mobile feedback service. First, we developed a metrics called degree of reliability (dor) (cf. Fig. 19). The latter is calculated for 


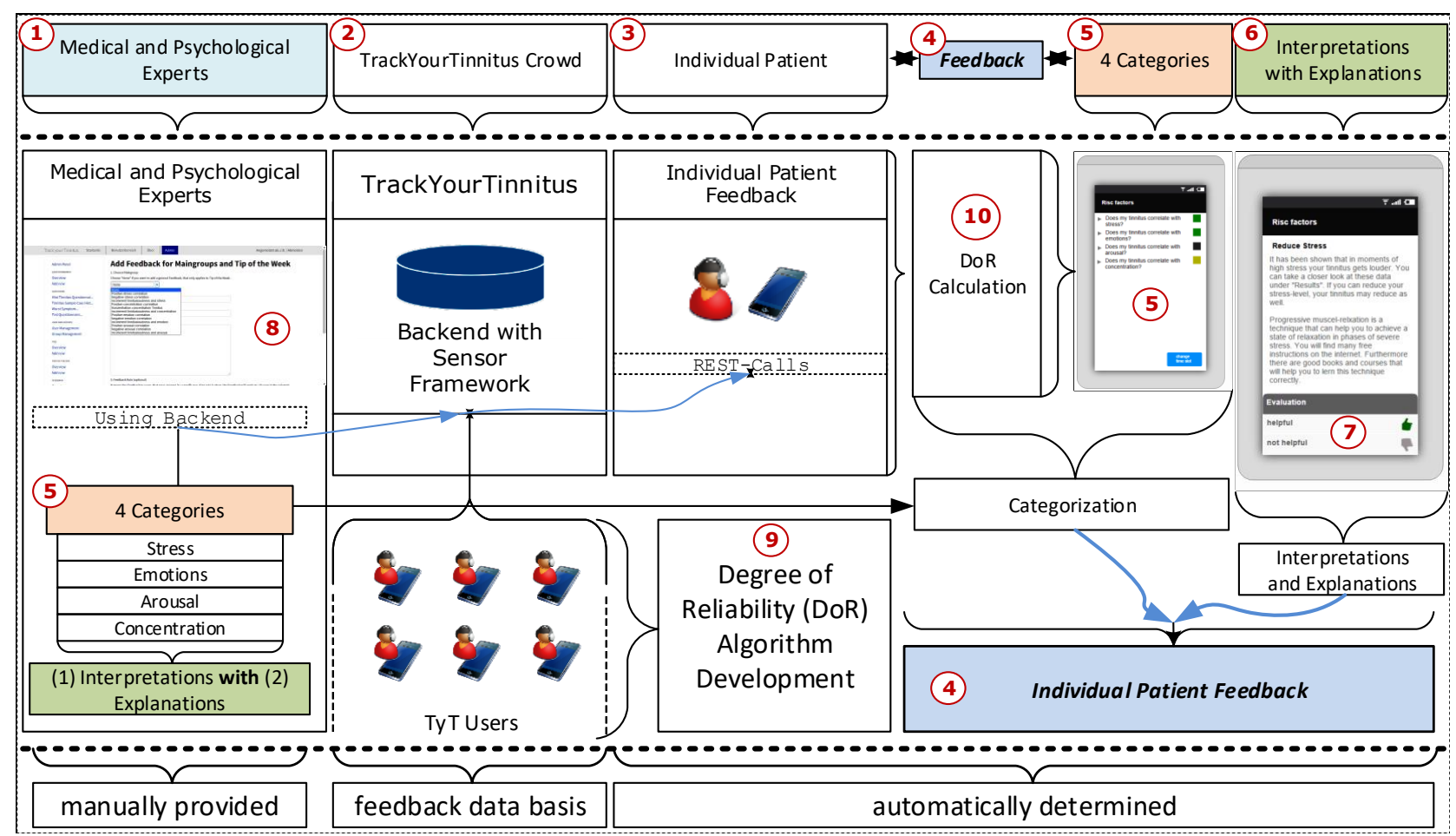

Figure 1: Patient Feedback Overview

each feedback category, indicating whether the amount of collected patient data is sufficient. To evaluate sufficiency for an individual patient, we considered collected data of all TYT users. Thereby, different perspectives were considered. For example, we calculated dor for category stress and related it to the amount of user notifications (cf. Fig. 2). Based on this, we developed a scale for dor as depicted in Fig. 4(3). Note that $d o r \geq 0.6$ must hold in order to start calculating the correlation for a category. For example, if the calculated dor for category stress is less than 0.6, the calculation needed for deciding whether the patient belongs to this category will not be started. If there are not enough data for categorizing a user, the TYT mobile feedback service returns this information to TYT users. Note that each category is considered independently with respect to the needed amount of data. This allows patients to obtain direct and valuable feedback on their collected data. Either they unveil that not enough data was collected or that feedback evolves over time.

Second, the correlation of a category is based on the Pearson product-moment correlation coefficient (PCC) [13] (cf. Fig. 110). Recall that the degree of reliability (dor) is coupled with the correlation calculation such that $P C C$ is only calculated if $d o r \geq 0.6$ holds. To tie correlation calculation with the degree of reliability revealed two advantages: First, patients may compare their assignments among the four categories. For example, if a patient belongs to category stress, but not to concentration, more data needs to be collected with respect to concentration (cf. Table I Question 7). Second, the dor scale is based on all patients. Therefore, individuals will benefit from collected data of all TYT users.

\section{B. Patient Perspective}

Another fundamental perspective on the TYT mobile feedback service is the one of the patient. Fig. 3 presents examples of feedback screens regarding the smart mobile device of a patient. Additionally, the interactions between the screens are shown in Fig. 3. Patients use the feedback as follows: First, they click on My Feedback (cf. Fig. 3(1)). Second, after clicking on My Tinnitus, they configure the period of time for feedback calculation (cf. Fig. 3(2)). Note that Fig. 3(2) solely illustrates the specification of the start date for this calculation. Another screen is used for the end date. Third, the screen showing the categorization is presented to the mobile user (cf. Fig. 3(3)). Note that this screen is only presented if at least one degree of reliability of $\{$ mood, arousal, stress, concentration $\} \geq 0.6$ holds (cf. Figs. 3(3), 4(2)). In Fig. 3, for example, the result for category arousal indicates no correlation for the tinnitus of the respective patient, as the calculated degree of reliability is less than 0.6. Fourth, a patient may expand a category in order to get all interpretations assigned to this category (cf. Fig. 3(4)). If patients click on interpretations, the screen presented in Fig. 3(5) is displayed. Finally, patients may return feedback to the selected interpretation (cf. Fig. 38). 


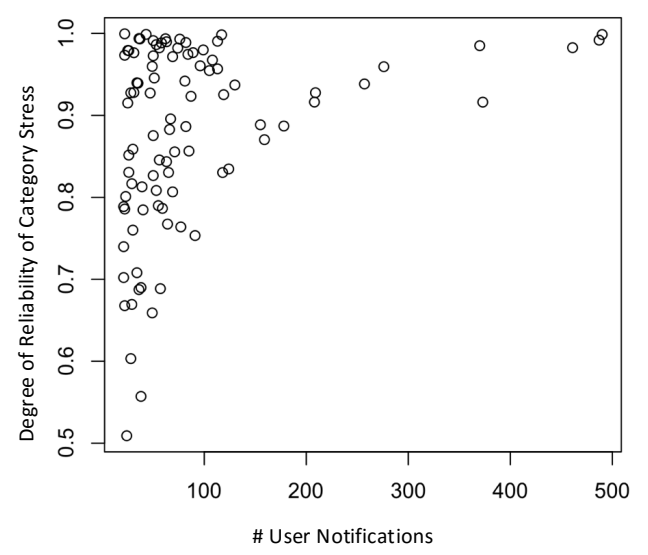

Figure 2: Degree of Reliability of Category Stress and User Notifications

\section{Technical Perspective}

We developed two algorithms for calculating proper user feedback. One of them determines the degree of reliability (dor), the other algorithm assigns patients to one of the four categories presented; e.g., if the tinnitus loudness correlates with stress of the respective user, the user will be assigned to the category stress. Note that both algorithms operate on the patient data gathered with the mobile assessment questionnaire. This data, in turn, is captured by entity standardanswers (cf. Table II).

As shown in Table II, entity standardanswers comprises 17 attributes. In the context of the two algorithms, several of these attributes are considered: First, attributes question 2 and question4-question 7 are used to calculate the degree of reliability as well as the assignment of users to categories. Second, attribute $u s e r \_i d$ represents the particular user for whom the feedback shall be calculated. Third, attribute created_at represents the date the assessment questionnaire was stored in the TYT database. Finally, the SQL command depicted at the bottom of Table II is used for calculating the patient feedback.

Prior to the feedback calculation (cf. Algorithm 2), the degree of reliability is determined by Algorithm 1. The decision which degree of reliability will be calculated by this algorithm is based on input parameter correlation. For example, if correlation has value 'Stress', the degree of reliability for category stress is calculated. Furthermore, input parameter standardanswers is used. Note that parameters loudness and tocorrelatewith are important as they constitute the two dimensions the degree of reliability is calculated for. Following this, tocorrelatewith may have values mood, arousal, stress, concentration. Based on these considerations, Algorithm 1 calculates the degree of reliability as follows (cf. Algorithm 1, Lines 17-29):

1) Sort array loudness in ascending order.

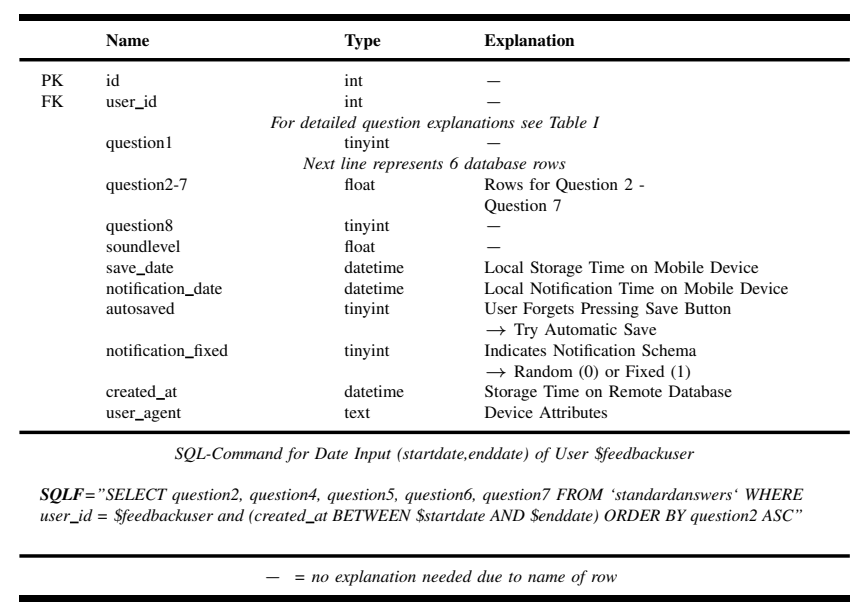

Table II: Entity standardanswers

2) Sort array tocorrelatewith according to the ordering of loudness. To identify corresponding entries, the primary key of entity standardanwers is used.

3) Split array loudness into two subarrays of equal length. The first subarray comprises all loudness elements of standardanswers with even index number; the second one, the elements with uneven index number.

4) Split array tocorrelatewith into two arrays of equal length.

5) Note that Steps 1 to 4 became necessary to ensure that the variance among the subarrays is equal from a statistical point of view.

6) Calculate Pearson product-moment correlation coefficients (PCC) [13], [14] $]^{1}$ : The first PCC is calculated based on the first subarray of loudness and the corresponding subarray of tocorrelatewith. The second PCC, in turn, is calculated based on the second subarray of loudness and the corresponding subarray tocorrelatewith.

7) Normalize the results by adding 1 to both PCCs; i.e., ensure that the result of the next calculation will be between 0 and 1.

8) Evaluate which PCC has a higher value and divide the lower PCC by the higher PCC to ensure normalization. Note that the result of the division takes two PCCs into account and hence establishes the degree of reliability between arrays loudness and tocorrelatewith.

9) Store division result to variable dor $\in[0,1]$.

The scale to evaluate degree of reliability values is shown in Fig. 4(3). Values below 0.6 indicate that not enough data for the respective category exist. Accordingly, the respective patient gets the feedback that not enough data has been gathered so far (cf. Fig. 3, Category arousal). In turn, values above 0.6 indicate that enough data has been

\footnotetext{
${ }^{1} \mathrm{PCC}$ represents a common way to calculate a correlation of two sets.
} 


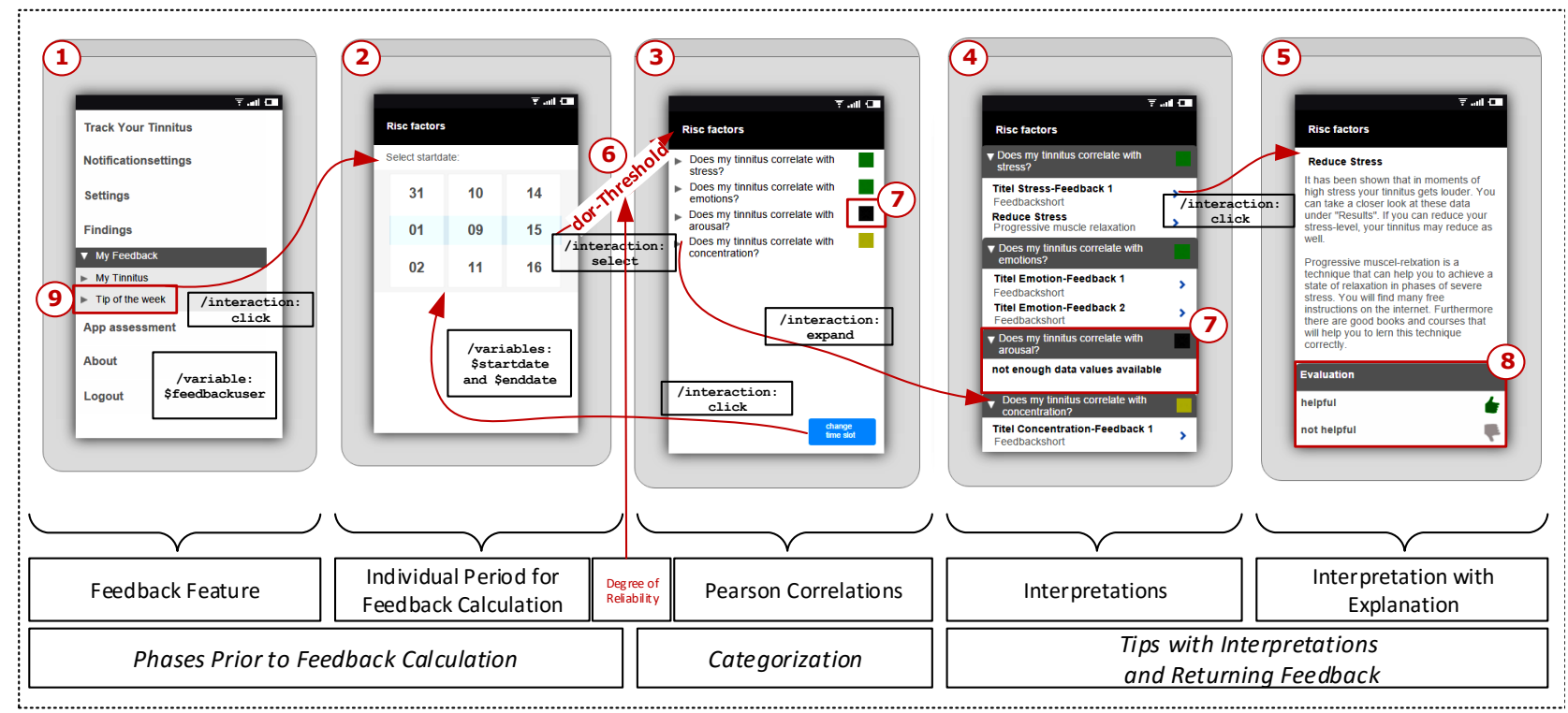

Figure 3: Patient Feedback Interaction

gathered. Altogether, first results indicate that the developed degree of reliability is appropriate for feedback calculation. Fig. 4 summarizes the basic steps for degree of reliability calculation. Algorithm 2, in turn, utilizes Algorithm 1 and assigns users to the four presented categories:

1) Load all relevant data from the database using the SQL command $S Q L F$ (cf. Table II).

2) Calculate the four required degrees of reliability (dors) with Algorithm 2 (cf. Lines 4 to 7).

3) If at least one dor is above 0.6, start feedback calcuation (cf. Fig. 4(2)).

4) Calculate the PCCs for those categories with a dor above 0.6. Otherwise set the respective PCC to -1 ; i.e., return feedback to patients that not enough data has been gathered (cf. category arousal in Fig. 3).

Note that the evaluation of all patient data revealed that a least 15 assessment questionnaires need to be completed to be able to provide valuable feedback (cf. Algorithm 2, Line $3)$.

\section{RELATED WORK}

In general, mobile crowdsensing is an emerging research topic in various application domains [15], [16]. Interestingly, in the medical domain, mobile crowdsensing applications have been less proposed so far. One reason that the medical domain is less considered might be related to legal and data privacy issues [17]. However, using mobile crowdsensing in the medical context is promising [18], since mobile crowdsensing has unique features to gather valuable data [19]. In particular, mobile crowdsensing may gather contextaware [20] as well as daily life data [21] more effectively. Altogether, mobile crowdsensing is an emerging topic. The utilization of its possibilities, in turn, is still at the beginning.
Mobile systems offer also opportunities to measure behavioral or physiological data in daily life [22]. In this context, EMA approaches are considered to offer unprecedented

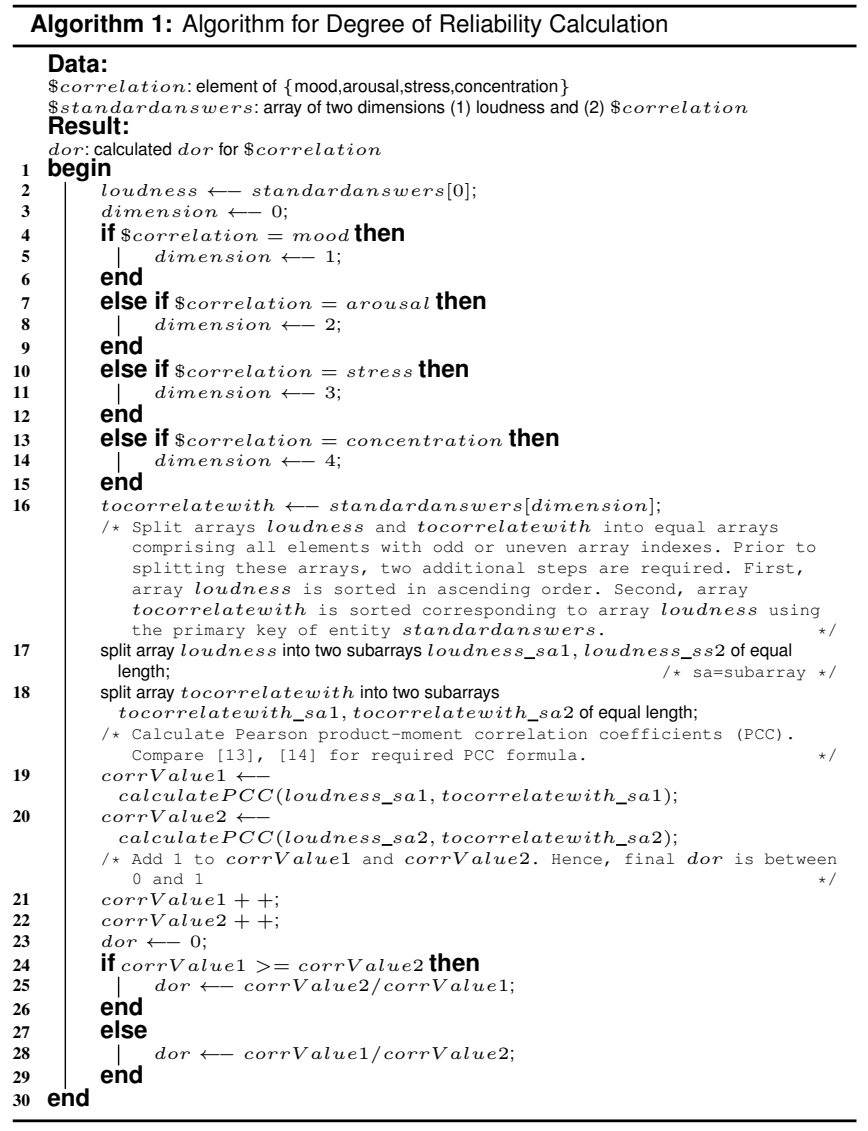




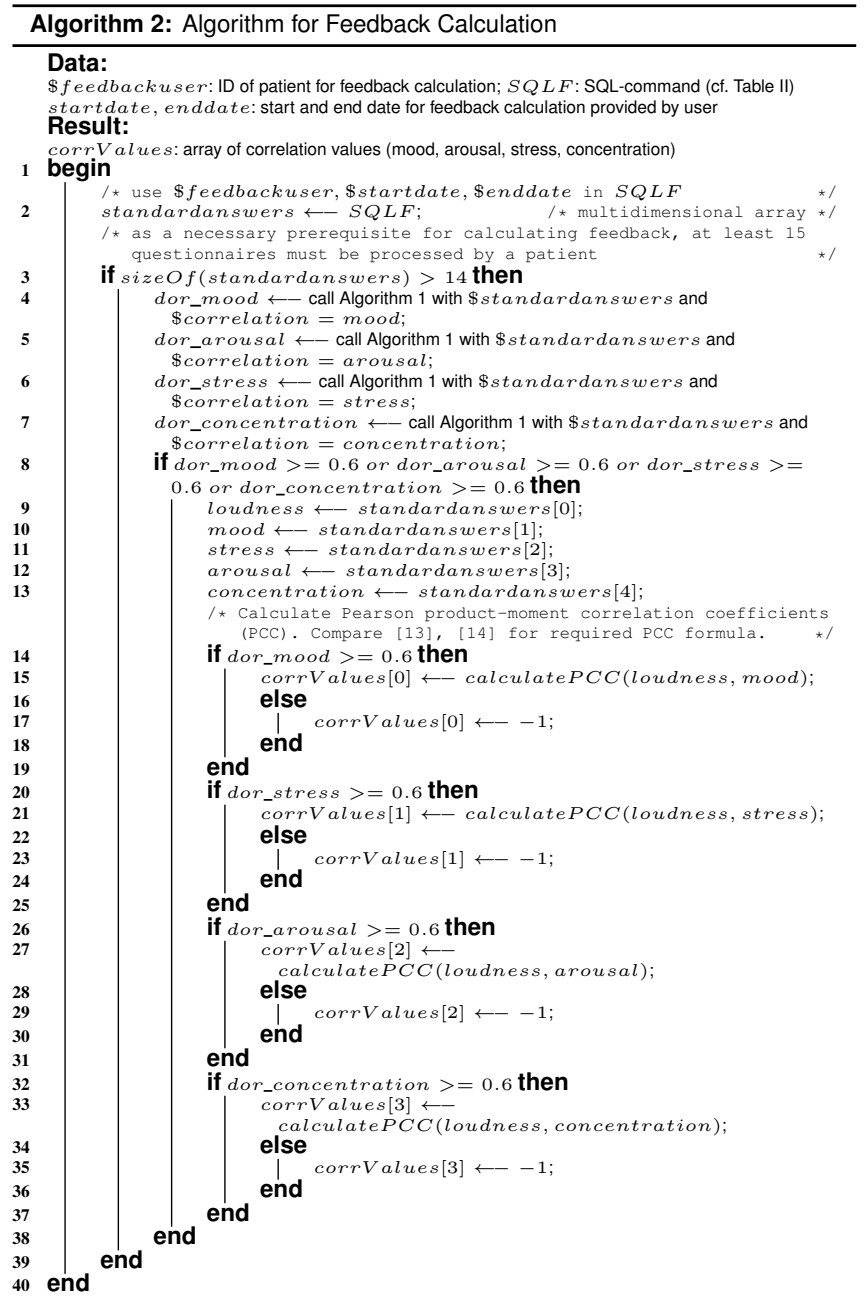

opportunities to study neuropsychiatric symptoms under ecologically valid conditions [23]. Besides TrackYourTinnitus, two further studies, namely [24] as well as [25], presented EMA approaches to track tinnitus in daily life.

Moreover, there exist approaches enabling immediate mobile feedback based on personally gathered data. In general, data sensing with smart mobile devices offers new ways to support mobile users in various scenarios [5]. In the context of personalized healthcare, for example, many patients crave for immediate feedback. Furthermore, patients expect feedback directly provided to their smart mobile device [12], [26]. In this context, [27] presents a mobile application that provides patients with valuable information for their daily insulin dosages. Based on previous dosages, in combination with context data, the application indicates whether the current situation is similar to previously recorded situations. A broader perspective for combining personal data gathered with mobile devices with context information is presented in [28]. The latter describes feedback as a crucial incentive to increase patient motivation. Finally, [29] presents mobile

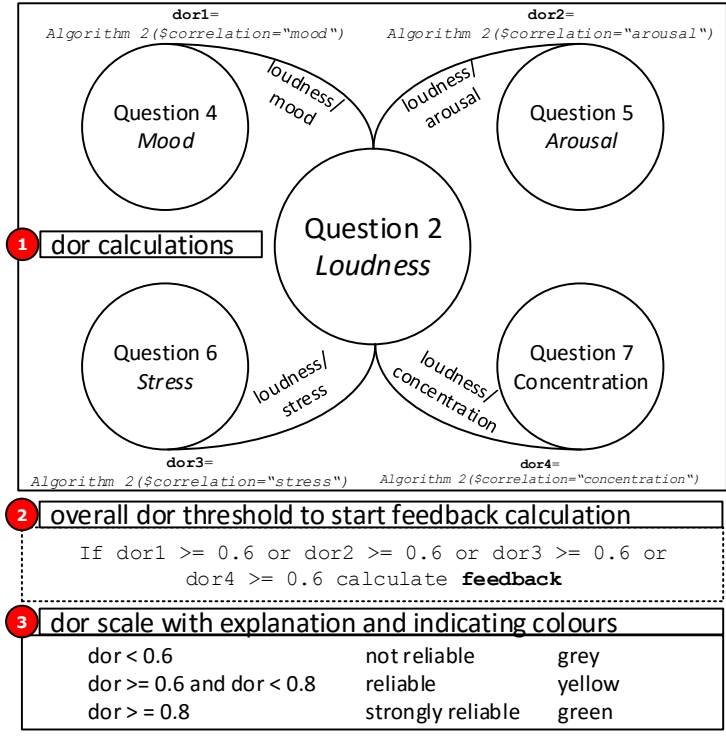

Figure 4: Degree of Reliability Calculation

applications that systematically measure vital signs enabling immediate feedback to users. Overall, the use of longitudinal patient data, gathered with a mobile crowdsensing service, for providing immediate feedback has been less considered by other approaches so far. To conclude, in various life domains, the feasibility of mobile crowdsensing has proven its usefulness. The medical field, albeit a highly promising application for mobile crowdsensing approaches, has been neglected so far.

\section{Summary AND OUTLOOK}

Using mobile crowdsensing offers promising perspectives for tinnitus assessment, therapy and research as well as for the medical field in general. With TYT, we obtained results that allow for totally new insights regarding tinnitus variability. The results further provide the basis for developing novel mobile crowdsensing services that foster tinnitus assessment, therapy, and research. In this context, we presented the patient feedback service we developed. In particular, we described a method to identify patient subgroups. Note that required data for such identification could not have been gathered without using mobile crowdsensing services.

The feedback service was integrated with the TYT backend as well as the Android mobile application. In future work, we will integrate the feedback service with the iOS mobile application as well. Furthermore, we will enhance the feedback service. We are working on techniques that allow medical experts to flexibly create feedback rules on their own. However, the feedback services already indicate that users are actually motivated to use this novel service. Notably, still more incentives and features are required to increase user motivation and hence to gather more valuable data on the tinnitus disease. In order to provide even more 
valuable feedback to users, medical experts as well as researchers are working on novel algorithms to automatically evaluate patient data. Altogether, over the next few years, mobile crowdsensing services will become increasingly important for collecting large and ecological valid longitudinal datasets in the context of clinical research.

\section{REFERENCES}

[1] B. Langguth, "A review of tinnitus symptoms beyond'ringing in the ears': a call to action," Current Medical Research \& Opinion, vol. 27, no. 8, pp. 1635-1643, 2011.

[2] M. Landgrebe et al., "The tinnitus research initiative (tri) database: a new approach for delineation of tinnitus subtypes and generation of predictors for treatment outcome," $B M C$ medical informatics and decision making, vol. 10, no. 1, p. 42, 2010 .

[3] C. Cederroth, B. Canlon, and B. Langguth, "Hearing loss and tinnitus [mdash] are funders and industry listening?" Nature biotechnology, vol. 31, no. 11, pp. 972-974, 2013.

[4] W. Schlee et al., "Measuring the moment-to-moment variability of tinnitus: the trackyourtinnitus smart phone app," Frontiers in Aging Neuroscience, vol. 8, 2016.

[5] N. Lane et al., "A survey of mobile phone sensing," IEEE Communications Magazine, vol. 48, no. 9, pp. 140-150, 2010.

[6] R. Pryss et al., "Mobile crowd sensing services for tinnitus assessment, therapy, and research," in Int'l Conf on Mobile Services. IEEE, 2015, pp. 352-359.

[7] R. Ganti, F. Ye, and H. Lei, "Mobile crowdsensing: current state and future challenges," Communications Magazine, IEEE, vol. 49, no. 11, pp. 32-39, 2011.

[8] T. Probst et al., "Emotion dynamics and tinnitus: Daily life data from the "trackyourtinnitus" application," Scientific reports, vol. 6, 2016.

[9] —-, "Outpatient tinnitus clinic, self-help web platform, or mobile application to recruit tinnitus study samples?" Frontiers in Aging Neuroscience, vol. 9, p. 113, 2017.

[10] T. Trull and U. Ebner-Priemer, "Ambulatory assessment," Annual review of clinical psychology, vol. 9, pp. 151-176, 2013.

[11] R. Pryss et al., "Mobile crowd sensing in clinical and psychological trials-a case study." IEEE, 2015, pp. 23-24.

[12] C. Freifeld et al., "Participatory epidemiology: use of mobile phones for community-based health reporting," PLoS Med, vol. 7 , no. 12,2010 .

[13] K. Pearson, "Note on regression and inheritance in the case of two parents," Proceedings of the Royal Society of London, pp. 240-242, 1895.

[14] S. Stigler, "Francis galton's account of the invention of correlation," Statistical Science, pp. 73-79, 1989.
[15] L. Shu, Y. Chen, Z. Huo, N. Bergmann, and L. Wang, "When mobile crowd sensing meets traditional industry," IEEE Access, 2017.

[16] H. Li, T. Li, and Y. Wang, "Dynamic participant recruitment of mobile crowd sensing for heterogeneous sensing tasks," in 12th International Conference on Mobile Ad Hoc and Sensor Systems. IEEE, 2015, pp. 136-144.

[17] D. Christin et al., "A survey on privacy in mobile participatory sensing applications," Journal of Systems and Software, vol. 84, no. 11, pp. 1928-1946, 2011.

[18] R. Ganti, F. Ye, and H. Lei, "Mobile crowdsensing: current state and future challenges," IEEE Communications Magazine, vol. 49, no. 11, 2011.

[19] M. Demirbas et al., "Crowd-sourced sensing and collaboration using twitter," in International Symposium on a World of Wireless Mobile and Multimedia Networks. IEEE, 2010, pp. $1-9$.

[20] H. Ma, D. Zhao, and P. Yuan, "Opportunities in mobile crowd sensing," IEEE Communications Magazine, vol. 52, no. 8, pp. 29-35, 2014.

[21] T. Probst et al., "Emotional states as mediators between tinnitus loudness and tinnitus distress in daily life: Results from the "trackyourtinnitus" application," Scientific reports, vol. 6, 2016.

[22] U. Ebner-Priemer and T. Kubiak, "Psychological and psychophysiological ambulatory monitoring," European Journal of Psychological Assessment, vol. 23, no. 4, pp. 214-226, 2007.

[23] I. Myin-Germeys et al., "Experience sampling research in psychopathology: opening the black box of daily life," Psychological medicine, vol. 39, no. 9, p. 1533, 2009.

[24] M. Wilson et al., "Ecological momentary assessment of tinnitus using smartphone technology a pilot study," Otolaryngology-Head and Neck Surgery, p. $0194599815569692,2015$.

[25] J. Henry et al., "Pilot study to evaluate ecological momentary assessment of tinnitus," Ear and hearing, vol. 32, no. 2, p. 179, 2012.

[26] L. Moser and P. Melliar-Smith, "Personal health monitoring using a smartphone," in Int'l Conf on Mobile Services. IEEE, 2015, pp. 344-351.

[27] D. Preuveneers and Y. Berbers, "Mobile phones assisting with health self-care: a diabetes case study," in Proc 10th Int'l Conf on Human computer interaction with mobile devices and services. ACM, 2008, pp. 177-186.

[28] P. Klasnja and W. Pratt, "Healthcare in the pocket: mapping the space of mobile-phone health interventions," Journal of biomedical informatics, vol. 45, no. 1, pp. 184-198, 2012.

[29] M. Morris and F. Guilak, "Mobile heart health: project highlight," Pervasive Computing, vol. 8, no. 2, pp. 57-61, 2009. 